\title{
Characterisation of $\mathbf{L}$. monocytogenes strains isolated from salad vegetables
}

\begin{abstract}
Seventy-one strains of L. monocytogenes isolated from raw salad vegetables consumed in the minimally processed state in Malaysia were characterised by serotyping, random amplified polymorphic DNA-polymerase chain reaction analysis (RAPD-PCR) and antimicrobial susceptibility testing. Based on serotyping, only one strain belonged to serotype 4 while the rest were from serotype 1. RAPD-PCR typing was carried out using two primers, i.e., OPAR8 and GEN 1-50-09. Based on RAPD-PCR fingerprinting, the strains could be grouped into six composite profile groups and a solitary isolate. The isolates were also evaluated against 16 antibiotics. Excluding nalidixic acid and oxacillin for which L. monocytogenes has natural resistance, the highest prevalence of resistance was against cephalexin. None of the isolates were resistant against imipenem and kanamycin. The multiple antibiotic resistance index (MARI) ranged from 0.06 to 0.63 . It was found that most $(82 \%)$ isolates were susceptible to antibiotics commonly used in veterinary and human listeriosis treatment.
\end{abstract}

Keyword: L. monocytogenes; Raw vegetables; Salad vegetables; Serotyping; RAPD; Random amplified polymorphic DNA; Polymerase chain reaction; PCR; Antimicrobial Susceptibility; Malaysia; Antibiotics; Antibiotic resistance 